\title{
Subtrochanteric Fracture In A Chinese Woman With Paget's Disease Of Bone And On Long Term Bisphosphonate Therapy: Could It Be An Insufficiency Fracture?
}

\author{
CK Lee, MBBS, P Jeremy, MS Orth (UM), s simmrat, MS Orth (UM), M Azura, MS Orth (UM) \\ Department of Orthopaedic Surgery, University Malaya Medical Centre, Kuala Lumpur, Malaysia
}

\begin{abstract}
Paget's disease is common in Western countries but is very rare in Chinese populations. Although bisphosphonate has been widely used to treat symptomatic Paget's disease, prolonged use may be associated with insufficiency fracture. We highlight this rare case of Paget's disease in a Chinese lady who presented with an insufficiency fracture following long-term use of bisphosphonate.
\end{abstract}

\section{Key Words:}

Paget's Disease, Chinese, Insufficiency Fracture, Bisphosphonate, Alendronate

\section{INTRODUCTION}

First described by Sir James Paget in 1876, Paget's disease otherwise known as osteitis deformans, is characterized by a focal increase in osteoclastic bone resorption followed by a compensatory increase in bone formation, resulting in disorganized lamellar bone, which is architecturally abnormal and mechanically weak. Although Paget's disease is common in Western countries, it is very rare in Chinese populations ${ }^{1}$. Alendronate, which is the third generation bisphosphonate, has been widely used to treat symptomatic Paget's disease. However, prolonged use of alendronate may be associated with insufficiency fracture ${ }^{2,3}$. We highlight this rare case of Paget's disease in a Chinese patient who presented with an insufficiency fracture following long-term treatment with bisphosphonate therapy.

\section{CASE REPORT}

A 64-year-old Chinese lady with underlying Paget's disease, presented to our Emergency Department with a fracture in the subtrochanteric region of the right femur following a trivial fall. There was no other injury noted. She had bone pain over her right thigh for the two months prior to her fall. The patient was diagnosed with Paget's disease of bone in 1998, when she presented with hearing impairment due to otosclerosis. Gradually, she also developed blurred vision, skull enlargement, facial changes and bowing of the right leg. She did not any have other medical problems and there was no family history of Paget's disease. There was no mixed marriage in her family. She had been treated with alendronate (Fosamax $\left.{ }^{\circledR}\right)$ since her diagnosis of Paget's disease.

Physical examination revealed a painful and deformed right thigh consistent with a fracture of the femur. There was no neurovascular injury. The patient exhibited the typical facial appearance of Paget's disease i.e. broadened forehead and enlarged skull (Fig. 1) as well as bowing of the right tibia. Plain radiograph of the pelvis showed a fracture at the subtrochanteric region with areas of patchy sclerosis and lucency (Fig. 2a). Skull x-rays showed features of typical Paget's disease with cotton wool appearance (Fig. 2b). Serum alkaline phosphatase levels were initially high, but normalized 5 months after her injury. She had normal serum calcium and phosphate levels.

The fracture was treated surgically with an intramedullary locking nail (Fig. 3). Histopathological examination of the intraoperatively obtained bone marrow revealed no malignant changes. The fracture healed well without any complications.

\section{DISCUSSION}

Although relatively common in Western countries, the incidence of Paget's disease of bone in the Chinese population is extremely rare ${ }^{1}$. Only 9 cases have been reported in the English literature and this is the second case reported in Malaysia at the time of writing this report ${ }^{1}$. The first case was reported in 1955 by $\mathrm{Khoo}^{4}$. The reason for the distinctive distribution of Paget's disease in different ethnic groups and geographic regions is still unclear ${ }^{1}$.

Most patients are diagnosed incidentally. When symptomatic, patients usually present with bone pain or bony deformity (i.e. skull enlargement, broadening of face or jaw and bowing of the leg). In our report, this patient presented with pathological fracture of femur. In Paget's disease, as a result of abnormal osteoclastic bone resorption 


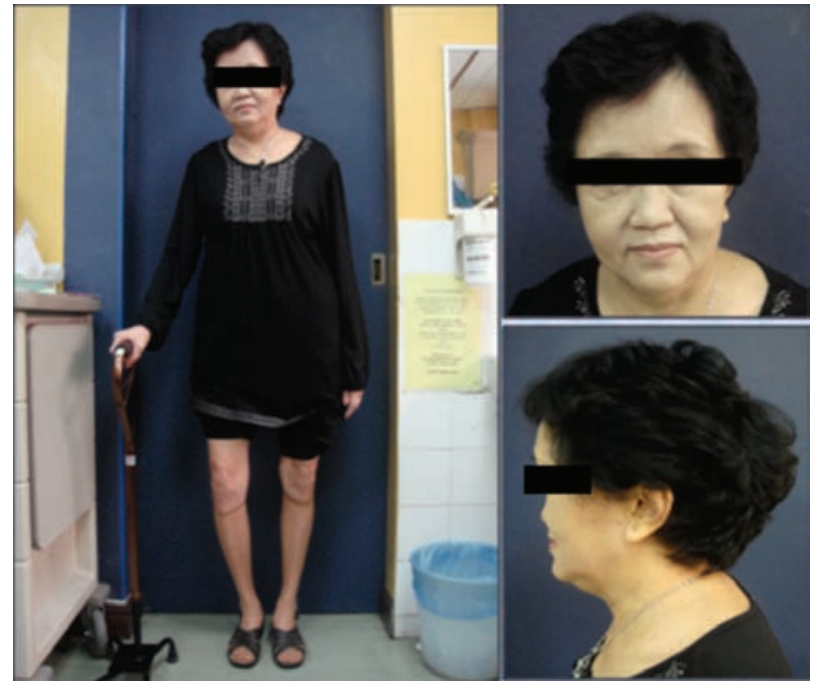

Fig. 1: Clinical photograph (taken postoperatively) showed bowed leg, skull enlargement, broadened forehead and swelling of right Zygomatic bone causing facial asymmetry.

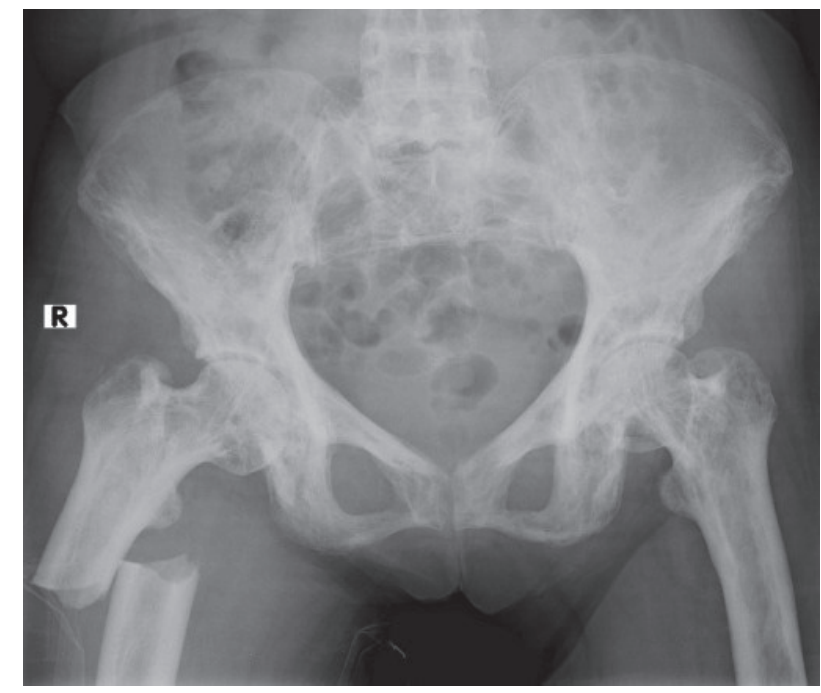

Fig. 2a: Pelvis radiograph showed areas of patchy sclerosis and lucency over the pelvis and its pathological fracture of subtrochanter right femur.

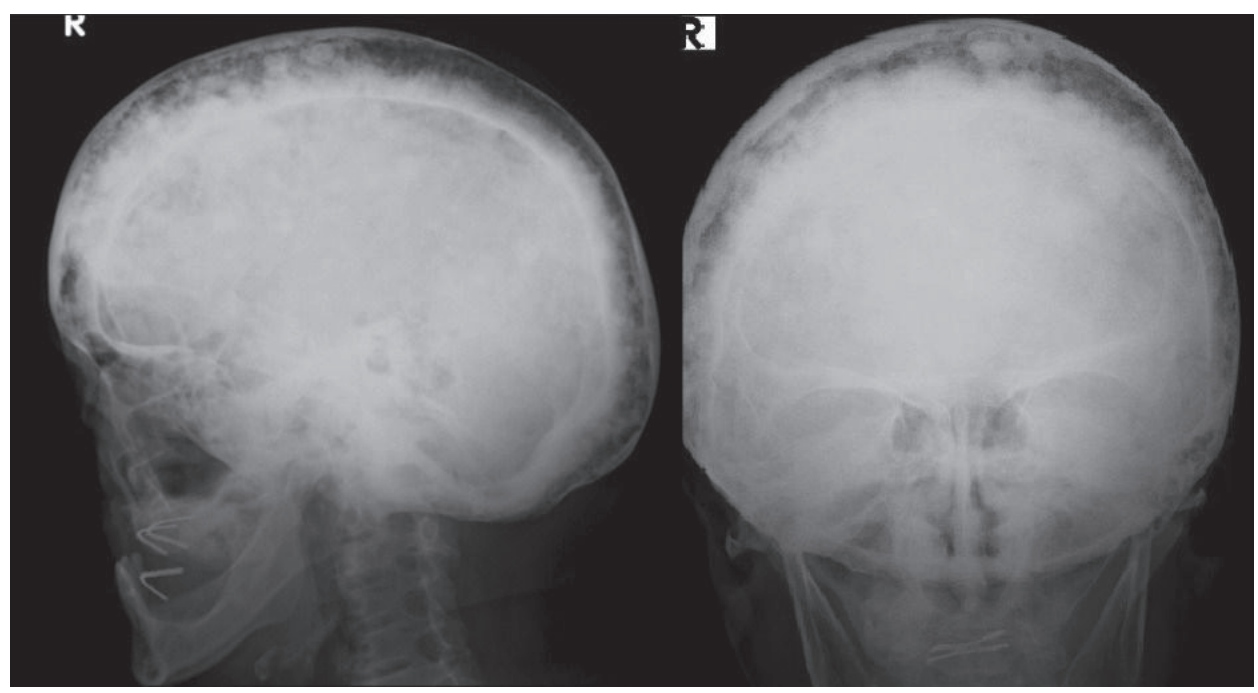

Fig. 2 b: Skull radiograph showed the pathognomonic cotton wool appearance.

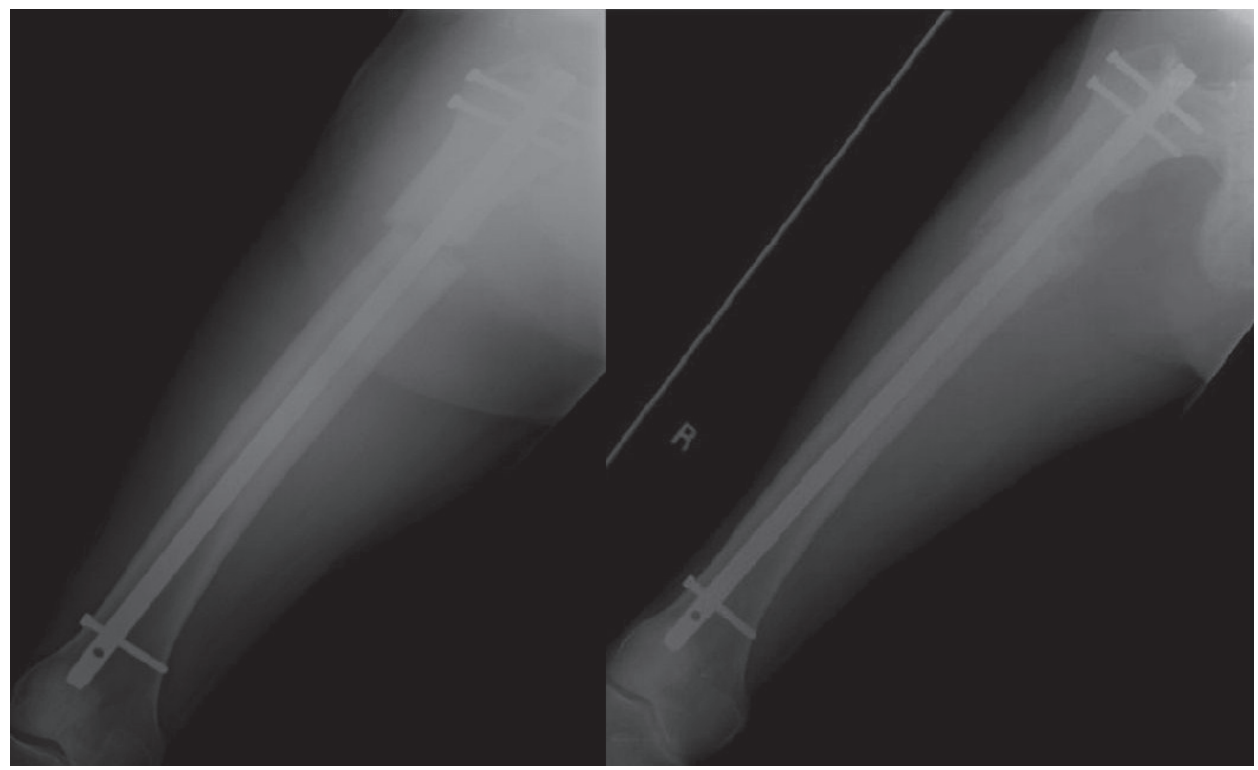

Fig. 3: Radiography of right femur at 2 days and 5 months after intramedullary locking nail. 
and disorganised formation of new lamellar bone, the bone becomes brittle. This led to the patient experiencing right thigh pain prior to her fall. She was not able to differentiate whether the trivial fall caused her fracture or the fracture led to her fall.

Pathological femur fracture is not uncommon in Paget's disease. When it does occur, the fracture usually occurs at the neck of femur because biomechanically, it is the weakest region of the bone. Pathological fracture at the subtrochanteric region is rare, because it is the strongest region of the femur. As this patient had also been treated with long-term alendronate (12 years), this diaphysealmetaphyseal junction fracture of the femur may be associated with the new form of insufficiency fracture postulated by Goh et al and Andrew et al ${ }^{2,3}$. Both Goh and Andrew have suggested that prolonged use of alendronate leads to diminished osteoclast activity, reduced bone remodelling and thus impaired microdamage repair at stressloading sites, which in this case was the subtrochanteric region. This gradually weakens the bone and leads to fracture.

Diagnosis of Paget's disease is usually made based on clinical features and pathognomonic radiological findings ${ }^{5}$. This patient exhibited the typical facial appearance of Paget's disease (Fig. 1) as well as classical radiological findings (Fig. 2a and 2b). Serum laboratory testing is of very limited value in making the diagnosis. Alkaline phosphatase (ALP), which rises in Paget's disease, is usually used as the biochemical marker to monitor disease progress and response to medication such as bisphosphonate. As this patient had been treated with alendronate, her ALP was expected to be normal. The initially increased ALP was actually due to the fracture. Bone scans typically show low specificity in terms of diagnosis of Paget's disease; thus it is only recommended for monitoring and evaluating the extent of the disease. Malignant transformation is the most serious complication of Paget's disease with osteosarcoma being the most common malignancy arising in the disorder. In the present case, histopathology examination of the bone specimen ruled out malignant changes.

The natural history of Paget's disease described in the standard orthopaedic textbooks and references is based on the study on the Western population. As described by Wang, although the clinical features of Paget's disease in Chinese population align closely to what is described for the Western population, one should not assume that the prognosis and clinical outcome of Paget's disease in Chinese population is also similar to the Western population as the number of reported cases of Paget's disease in Chinese has been very small ${ }^{1}$. Furthermore, Wang also noted that there is more often skull involvement and a higher frequency of monostotic cases and symptomatic cases observed in Chinese patients. The incidence of Paget's disease in Chinese population is probably underreported. Therefore, more reports and studies, especially genetic investigations, are needed in order to form a better understanding in Paget's disease among the Chinese population.

We hope that by highlighting this extremely rare condition of Paget's disease in the Chinese population, the rare presentation of insufficiency fracture due to long term bisphosphonate therapy and the association of both conditions, we have created a learning opportunity for orthopaedic surgeons and a point of interest for future research. 


\section{REFERENCES}

1. Wang WC, Cheng YS, Chen CH, Lin YJ, Chen YK, Lin LM. Paget's disease of bone in a Chinese patient: A case report and review of the literature. Oral Surg Oral Med Oral Pathol Oral Radiol Endod 2005; 99: 727-33.

2. Goh SK, Yang KY, Koh JS, Wong MK, Chua SY, Chua DT et al. Subtrochanteric insufficiency fractures in patients on alendronate therapy: A caution. J Bone Joint Surg [Br] 2007; 89: 349-53.

3. Neviaser AS, Lane JM, Lenart BA, Edobor-Osula F, Lorich DG. Low-energy femoral shaft fractures associated with alendronate use. J Orthop Trauma 2008; 22(5): 346-50.

4. Khoo FY. Paget's disease in the Chinese. Case report. Med J Malaya 1955; 9: 281-3.

5. Hosking D, Meunier PJ, Ringe JD, Reginster JY, Gennari C. Paget's disease of bone: diagnosis and management. BMJ 1996; 312: 491-4. 